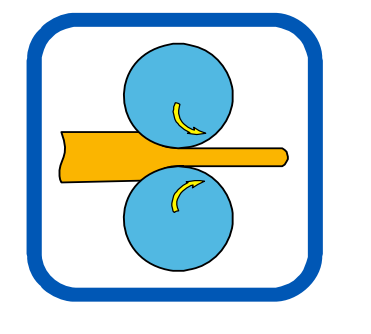

УДК 539.37

\section{РАЗДЕЛ III \\ ПРОЦЕССЫ ОБРАБОТКИ ДАВЛЕНИЕМ \\ В МЕТАЛЛУРГИИ}

DOI: $10.37142 / 2076-2151 / 2019-2(49) 150$

Медведев М. И. Андреев В. В. Фролов Я. В. Бобух А. С.

\title{
ОЦЕНКА СОПРОТИВЛЕНИЯ ДЕФОРМАЦИИ ПРИ ГОРЯЧЕМ ПРЕССОВАНИИ ТРУБ ИЗ СПЛАВОВ НА ОСНОВЕ НИКЕЛЯ
}

Известно [1-5], что величина сопротивления деформации является определяющей при расчетах силовых характеристик прессования труб, а также разогрева металла в очаге деформации.

Под сопротивлением деформации обычно подразумевается величина истинного предела текучести металла при соответствующей температуре, степени и скорости деформации, для определения которого в процессах ОМД широко используется «метод термомеханических коэффициентов» $[6,7]$. В указанном методе базисные значения сопротивления деформации получены при механических испытаниях при температуре металла $1000{ }^{\circ} \mathrm{C}$, степени деформации 0,1 и скорости деформации $10 \mathrm{c}^{-1}$, умножаются на термомеханические коэффициенты, учитывающие фактическую степень, скорость и температуру деформации металла. При этом расчетное значение сопротивления деформации определяется по формуле [8]:

$$
\sigma=k_{t} \cdot k_{\varepsilon} \cdot k_{u} \cdot \sigma_{0 S},
$$

где: $\sigma_{0 S}$ - базисное значение сопротивления деформации при вышеуказанных параметрах деформации; $k_{t} ; k_{\varepsilon} ; k_{u}$ - термомеханические коэффициенты: $k_{t}-$ температурный; $k_{\varepsilon}$ - деформационный; $k_{u}$ - скоростной.

Этот метод позволяет получить достоверные данные для процессов прокатки. Вместе с тем, для процесса горячего прессования труб, отличающегося схемой напряженного состояния - всесторонним неравномерным сжатием, степенью деформации $90 \%$ и более, температурой металла до $1250^{\circ}$ и скоростью деформации $100 \mathrm{c}^{-1}$, применение указанного метода для расчета усилий прессования не дает точных результатов.

В технической литературе существует значительное количество формул для расчета силы прессования как теоретических, так и экспериментальных, полученных на основании статистической обработки данных. Использование теоретических зависимостей весьма затруднено из-за сложностей в определении $\sigma_{\text {ист }}$, эмпирические формулы пригодны только для тех условий прессования, на основании которых они получены.

В связи с этим при расчетах силы прессования на трубопрессовых установках принято пользоваться обобщенной формулой, в виде [8]:

$$
P=F \cdot \ln \mu \cdot \rho,
$$

где $P$ - сила прессования, $\mathrm{MH} ; F$ - площадь поперечного сечения гильзы в подпрессовом состоянии, $\mathrm{M}^{2} ; \mu$ - коэффициент вытяжки при прессовании трубы; $\rho$ - коэффициент пропорциональности, включающий в себя сопротивление деформации, геометрию очага деформации и 
другие условия прессования. Его величина в той же мере зависит от химического состава стали, температуры, степени и скорости деформации, что и истинный предел текучести металла.

В дальнейшем величину $\rho$ для краткости будем называть сопротивлением деформации, которая определяется пересчетом из фактических замеров силы прессования. Основным преимуществом данного подхода к сопротивлению деформации является возможность достаточно простого его экспериментального определения.

В общем виде для процесса горячего прессования величина $\rho$ определяется по следующей зависимости [8]:

$$
\rho=\rho_{0} \cdot K_{T . S} \cdot K_{\mu} \cdot K_{\dot{\varepsilon}},
$$

где $\rho_{0}-$ сопротивление деформации при базовых условиях для каждой прессовой установки, МН/м²; $K_{T . S}$ - коэффициент, учитывающий отклонения температуры нагрева и толщины стенки гильз от базовых значений; $K_{\mu}-$ коэффициент, учитывающий отклонения степени деформации (коэффициентов вытяжки) от базовых значений; $K_{\dot{\varepsilon}}-$ коэффициент, учитывающий отклонения скорости деформации от базовых значений.

Целью работы является определение составляющих зависимости сопротивления деформации от химического состава материала, температуры, скорости и степени деформации, которые позволяют достаточно точно определить силовые параметры прессования труб из различных материалов.

Под базовыми условиями для различных прессовых установок принимается наиболее часто используемые при прессовании сплавов на основе никеля: температуру нагрева, толщину стенки гильз, диаметр втулки контейнера, а также время транспортных операций (табл. 1). При этом коэффициент вытяжки для этих условий составил 8, степень деформации $-87,5 \%$, скорость деформации $-90 \mathrm{c}^{-1}$.

Таблица 1

Базовые условия при прессовании сплавов на основе никеля

\begin{tabular}{|c|c|c|c|c|}
\hline $\begin{array}{c}\text { Усилие } \\
\text { прессовой } \\
\text { установки, МН }\end{array}$ & $\begin{array}{c}\text { Длительность } \\
\text { транспортных } \\
\text { операций, с }\end{array}$ & $\begin{array}{c}\text { Диаметр втулки } \\
\text { контейнера, мм }\end{array}$ & $\begin{array}{c}\text { Толщина } \\
\text { стенки } \\
\text { гиль3, мм }\end{array}$ & $\begin{array}{c}\text { Температура } \\
\text { нагрева } \\
\text { в печи, }{ }^{\circ} \mathrm{C}\end{array}$ \\
\hline 16,0 & 39 & 190 & 50 & 1150 \\
\hline 20,0 & 98 & 195 & 50 & 1200 \\
\hline 31,5 & 37 & 195 & 50 & 1150 \\
\hline 55,0 & 125 & 341 & 80 & 1200 \\
\hline
\end{tabular}

Известно, что сопротивление деформации сталей и сплавов зависит от степени их легирования [6]. Сопротивление деформации сплавов системы $\mathrm{Fe}-\mathrm{Ni}-\mathrm{Cr}$ постепенно возрастает по мере повышения в сплавах содержания Ni и Cr [9]. Данная зависимость получена на основе статистической обработки замеров силы прессования более 30 труднодеформируемых сплавов, таких как ХН32Т, ХН45Ю, ХН78Т, ХН40Б, Х23Н28МДТ и другие, при базовых условиях прессования: $\mathrm{D}_{\text {конт }}=195 \mathrm{Mм}, \mathrm{S}_{\text {гильзы }}=50 \mathrm{Mм}, \mathrm{T}=1150{ }^{\circ} \mathrm{C}$, коэффициент вытяжки $\mu=7 \ldots 10,0$ (рис. 1 ).

Данный график достаточно точно аппроксимируется выражением:

$$
\rho_{0}=200+1,2 \cdot(\mathrm{Ni}+\mathrm{Cr}, \%),\left[\mathrm{H} / \mathrm{MM}^{2}\right] .
$$

Введение в сплавы упрочняющих легирующих элементов, а именно $\mathrm{Mo}, \mathrm{W}, \mathrm{V}, \mathrm{Nb}$, дополнительно повышает сопротивление деформации матрицы пропорционально их процентному содержанию.

В общем виде эту зависимость можно представить следующим образом:

$$
\rho_{0}=200+1,2 \cdot(N i+C r, \%)+\alpha \cdot(M o+W+V+N b, \%),\left[\mathrm{H} / \mathrm{Mm}^{2}\right],
$$

где $\alpha$-размерный коэффициент для прессовых установок. 
При прессовании труб из вышеуказанных сплавов в диапазоне размеров Ø76...159 и толщиной стенки $S=10 \ldots 20$ мм на прессах $16,0 \mathrm{MH}, 31,5 \mathrm{MH}$ и 44,0 MН; труб Ø76...168 и толщиной стенки $S=10 \ldots 20$ мм на прессе $20 \mathrm{MH}$; Ø133...245 и толщиной стенки $S=14 \ldots 30$ мм на прессе $55 \mathrm{MH}$, экспериментально установлено, что величина коэффициента пропорциональности $(\alpha)$ составляет 2,0 при суммарном содержании $(M o+W+V+N b, \%)$ до $10 \%$ и $\alpha=6,5$ при суммарном содержании элементов более $10 \%$, что объясняется дополнительным упрочнением металла за счет образования избыточных фаз.

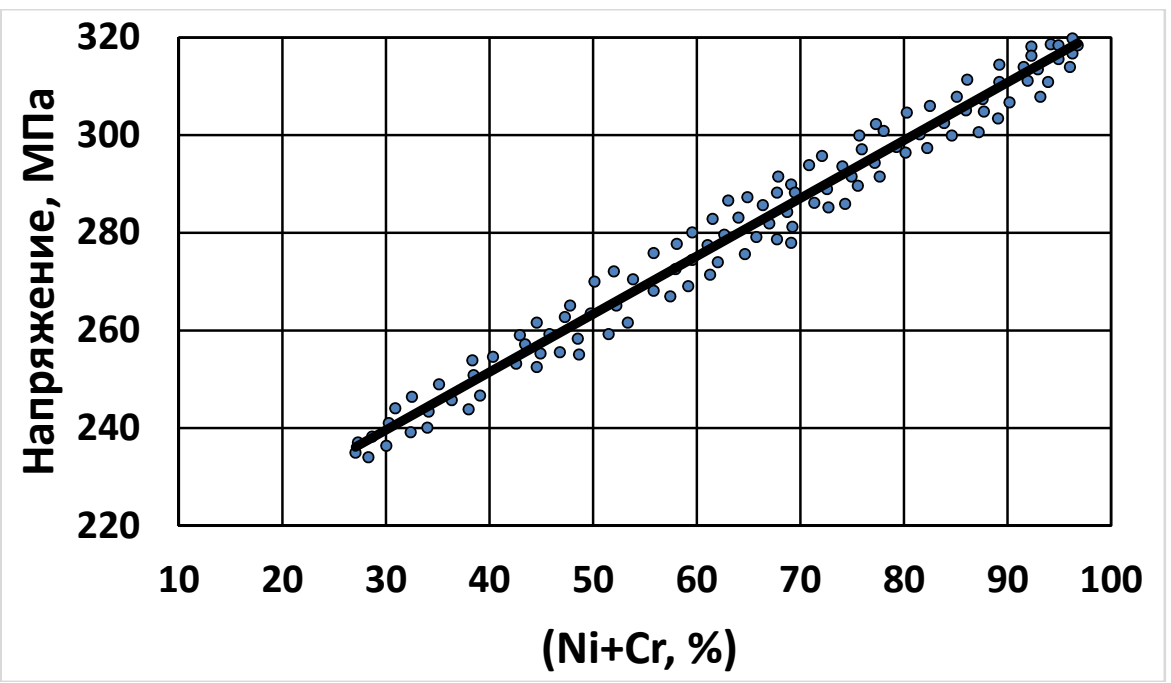

Рис. 1. Зависимость сопротивления деформации $\rho_{0}$ от процентного содержания легирующих элементов $(\mathrm{Ni}+\mathrm{Cr}, \%)$.

Полученные по формуле (5) значения сопротивления деформации отклоняются от фактических в пределах $10 \mathrm{MH} / \mathrm{M}^{2}$.

Наибольшее существенное влияние на сопротивление деформации оказывает температура металла, зависимость от которой по мнению многих авторов экспоненциальная [6]:

$$
\rho=\rho_{0}+\mathrm{e}^{\mathrm{m} \cdot\left(\mathrm{T}_{0}-\mathrm{T}_{\mathrm{H}}\right)-\Delta \mathrm{t}_{0}+\Delta \mathrm{t}_{\mathrm{S}, \mathrm{T}},}
$$

где $\rho_{0}-$ сопротивление деформации при базовых условиях для каждой прессовой установки, $\mathrm{MH} / \mathrm{M}^{2} ; \mathrm{T}_{0}$ - базовая температура нагрева гильзы для каждой прессовой заготовки, ${ }^{\circ} \mathrm{C}$; $\mathrm{T}_{\mathrm{H}}$ - температура нагрева гильз, ${ }^{\circ} \mathrm{C} ; \Delta \mathrm{t}_{0}$ - изменение температуры гильз в процессе охлаждения за время вспомогательных операций при базовых условиях для каждой прессовой заготовки; $\Delta \mathrm{t}_{\mathrm{S}, \mathrm{T}}$ - изменение температуры гильз в процессе охлаждения за время вспомогательных операций с учетом отклонения толщины стенки и температуры нагрева гильз от базовых условий.

Исходя из этого, коэффициент, учитывающий влияние температуры нагрева и толщины стенки в общем виде равен:

$$
K_{T . S}=\mathrm{e}^{\mathrm{m} \cdot\left(\mathrm{T}_{0}-\mathrm{H}_{\mathrm{H}}-\Delta \mathrm{t}_{0}+\mathrm{t}_{\mathrm{ST}}\right)},
$$

а для прессовых установок (табл. 1) имеет следующий вид:

- для прессовых установок 16,0 MH, 31,5 MH:

$$
K_{T . S}=\mathrm{e}^{0,87 \cdot \mathrm{m} \cdot\left(\frac{4146}{\mathrm{~S}}+1065-\mathrm{T}_{\mathrm{H}}\right),}
$$

- для прессовых установок 20,0 MH:

$$
K_{T . S}=\mathrm{e}^{0,72 \cdot \mathrm{m} \cdot\left(\frac{8330}{\mathrm{~s}}+1036-\mathrm{T}_{\mathrm{H}}\right),}
$$

- для прессовых установок 55,0 MH: 


$$
K_{T . S}=\mathrm{e}^{0,76 \cdot \mathrm{m} \cdot\left(\frac{11320}{\mathrm{~s}}+1053-\mathrm{T}_{\mathrm{H}}\right)} .
$$

Определённые трудности существуют в определении коэффициента «т», так как различные авторы определяют его в разных условиях деформации. В табл. 2 приведены значения коэффициентов «т» по результатам исследований различных авторов.

Как видно из данных табл. 2, разброс значений «т» весьма значителен, что зависит от условий экспериментов. Рекомендуемые авторами настоящей работы значения базируются на статистических материалах, которые получены на различных прессовых установках. Разбивка сплавов в таблице условна, однако общая тенденция весьма корректна, при этом погрешность в определении «т» для конкретного сплава не превышает 5 \%.

Таблица 2

Значения коэффициента «т»

\begin{tabular}{|l|c|c|c|}
\hline \multicolumn{1}{|c|}{ Стали и сплавы } & \multicolumn{3}{|c|}{ Значения «т» } \\
\cline { 2 - 4 } & $\begin{array}{c}\text { A. В. Третьяков } \\
{[6]}\end{array}$ & $\begin{array}{c}\text { Шпиттель } \\
{[2]}\end{array}$ & $\begin{array}{c}\text { Рекомендуемые } \\
\text { авторами }\end{array}$ \\
\hline Нержавеющие аустенитные стали & 0,0028 & 0,00284 & 0,0040 \\
\hline Нержавеющие аустенитные стали с Mo & - & - & 0,0034 \\
\hline $\begin{array}{l}\text { Сплавы Ni-Cr-Fе при содержании } \\
\text { Ni = 20-45\% неупрочненные }\end{array}$ & - & 0,0022 & 0,0032 \\
\hline $\begin{array}{l}\text { Сплавы Ni-Cr-Fе с упрочняющими } \\
\text { элементами до 5\% }\end{array}$ & 0,0032 & 0,0023 & 0,0030 \\
\hline Сплавы Ni-Cr не упрочнённые & 0,0032 & 0,0023 & 0,0028 \\
\hline $\begin{array}{l}\text { Сплавы Ni-Cr с упрочняющими эле- } \\
\text { ментами до 10\% }\end{array}$ & - & 0,0020 & 0,0025 \\
\hline $\begin{array}{l}\text { Сплавы Ni-Cr с упрочняющими эле- } \\
\text { ментами более 10 \% }\end{array}$ & 0,0028 & 0,0030 & 0,0022 \\
\hline
\end{tabular}

В начале, до достижения степени деформации 30-35 \% идет непрерывный рост сопротивления деформации. После достижения $\rho_{\max }$ начинается резкое снижение $\rho$ в результате влияния разогрева металла. Это снижение заканчивается при достижении $\mu=15$, после чего сопротивление деформации не зависит от степени деформации (рис. 2).

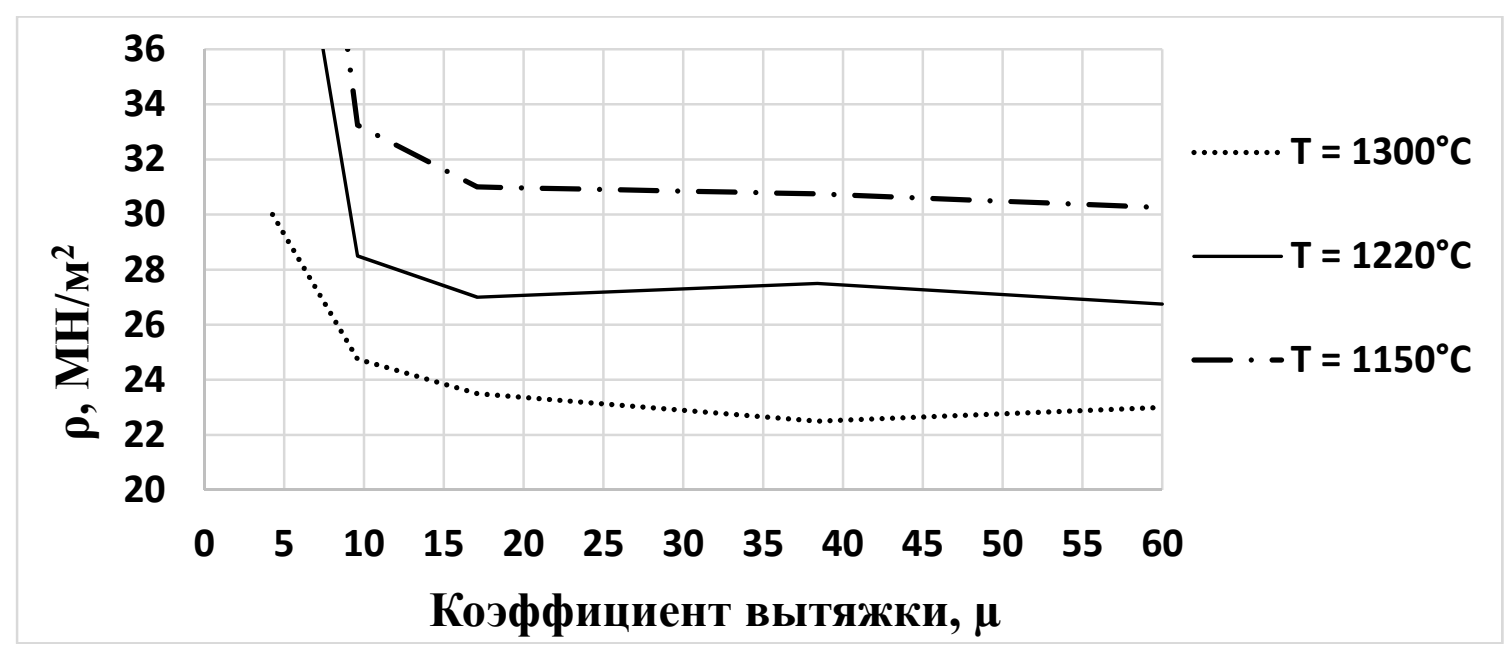

Рис. 2. Изменение $\rho$ в зависимости от коэффициента вытяжки $\mu$ при прессовании труб из стали $12 \mathrm{X} 18 \mathrm{H} 10 \mathrm{~T}$ 
В диапазоне коэффициентов вытяжки $\mu=5-15$, изменение сопротивления деформации (модуля прессования) не превышает $10 \mathrm{H} / \mathrm{mm}^{2}$ (5 \%). Если коэффициент вытяжки при базовых условиях равен 10 , то $\mathrm{K}_{\mu}=1$, при $\mu=5-15, \mathrm{~K}_{\mu}=1,05$, а при $\mu>15$, коэффициент $\mathrm{K}_{\mu}=0,95$.

Влияние скорости деформации на сопротивление деформации с достаточной точностью может быть представлено следующей зависимостью:

$$
\rho=\rho_{0} \cdot\left(\frac{\dot{\varepsilon}}{\dot{\varepsilon_{0}}}\right)^{\mathrm{n}},
$$

где $\rho_{0}-$ сопротивление деформации при базовых условиях, Н/мм²; $\dot{\varepsilon}$ и $\dot{\varepsilon_{0}}-$ скорость деформации, соответственно, для конкретных и базовых условий прессования, мм/c; n - показатель степени, значение которого определяется процессом упрочнения конкретного материала и определяется экспериментально.

Средняя скорость деформации при прессовании труб определяется упрощённой формулой Л. А. Шофмана [10]:

$$
\dot{\varepsilon}=\frac{6 \cdot \mathrm{U}_{\mathrm{cp}} \cdot \ln \mu}{\mathrm{D}_{\mathrm{k}}},
$$

где $\mathrm{U}_{\mathrm{cp}}$ - средняя скорость движения пресс-штемпеля, мм/c; $\mathrm{D}_{\mathrm{k}}$ - диаметр контейнера трубнопрофильного пресса, мм.

Прессование труднодеформируемых сплавов производится, как правило, при невысоких скоростях прессования $U_{c p}=100-200$ мм/с и в относительно узком диапазоне коэффициентов вытяжки 5...15. Для этих условий формулу (11) с учётом (12) можно представить в виде:

$$
\rho=\rho_{0} \cdot\left(\frac{\mathrm{D}_{\mathrm{k} 0}}{\mathrm{D}_{\mathrm{k}}}\right)^{\mathrm{n}}, \text { при этом } K_{\dot{\varepsilon}}=\left(\frac{\mathrm{D}_{\mathrm{k} 0}}{\mathrm{D}_{\mathrm{k}}}\right)^{\mathrm{n}},
$$

Показатели степени упрочнения «n» зависят от конкретного материала и определяется экспериментально. По данным работы [6] для нержавеющих сталей в горячем состоянии $\mathrm{n}=0,087$, для сплавов на основе никеля $\mathrm{n}=0,098$. Результаты обработки замеров усилий прессования на трубопрессовых установках $20 \mathrm{MH}$ и $55 \mathrm{MH}$ показали, что для труб из нержавеющих сталей и никелевых сплавов коэффициент $\mathrm{n}=0,18$. Такое различие в величине коэффициентов $\mathrm{n}$ с результатами работы $[2,6]$ объясняются более высокими степенями деформации при прессовании труб. Величина $\mathrm{n}=0,18$ для нержавеющих сталей подтверждается также экспериментальными данными.

\section{ВЫВОДЫ}

1. Определены все составляющие зависимости сопротивления деформации от химического состава материала, температуры, скорости и степени деформации, которые позволяют достаточно точно определить силовые параметры прессования труб из различных материалов.

2. Установлено, что величина коэффициента пропорциональности $(\alpha)$, который входит в уравнение для расчета сопротивления деформации при базовых условиях, составляет $\alpha=2,0$ при суммарном содержании $(M o+W+V+N b, \%)$ до $10 \%$ и $\alpha=6,5$ при суммарном содержании элементов более $10 \%$, что объясняется дополнительным упрочнением металла за счет образования избыточных фаз.

3. Определен показатель степени упрочнения «n» для условий прессования труб из нержавеющих сталей и никелевых сплавов на трубопрессовых установках $20 \mathrm{MH}$ и $55 \mathrm{MH}$, который составляет $\mathrm{n}=0,18$. 


\section{СПИСОК ИСПОЛЬЗОВАННОЙ ЛИТЕРАТУРЫ}

1. Манегин Ю. В., Притоманов А. Е., Шпиттель Т., Кнаушнер А. М. Горячее прессование труб и профилей. Москва : Металлургия, 1980. 272 с.

2. Хензель А., Шпиттель Т. Расчет энергосиловых параметров в процессах обработки металлов давления. Москва : Металлургия, 1982. 160 с.

3. Дыя Х., Бергеман Г. В., Соколов С. Ф., Андреев В. В. Экспериментальное исследование сопротивления деформации сталей и определение параметров упрочнения-разупрочнения при горячей обработке давлением. Обработка материалов давлением. 2015. № 2 (41). С. 211-217.

4. Клименко П. Л. Упрочнение стали и цветных металлов при холодной и горячей деформации: монография. Днепропетровск : Пороги, 2011. 187 с.

5. Фирсова Т. И. Методика оценки параметров упрочнения-разупрочнения реологически сложных металлов по пластометрическим кривым. Обработка материалов давлением. 2012. № 1 (3). С. 65-68.

6. Третьяков А. В., Зюзин В. Н. Механические свойства металлов и сплавов при обработке давлением. Москва : Металлургия, 1973. 224 с.

7. A. Lotkov., Grishkov V., Baturin A., et al. Yield stress and reversible strain in titanium nickelide alloys after warm abc pressing. Materials. 2019. 3258. 12. pp. 1-14. DOI: http://www.doi:10.3390/ma12193258

8. Медведев М. И., Беспалова Н. А., Царьков А. К. и др. Методика определения основных параметров прессования труб из трудно деформируемых сталей и сплавов. Металлургическая и горнорудная промышленность. 2007. № 3. С. 56-59.

9. Пластическая деформация сталей и сплавов. Сб. научн. тр. МИСиС. Москва : МИСиС, 1996. 460 с.

10. Шофман Л.А. Основы расчета процессов штамповки и прессования. Москва : Машгиз, 1961. 399 с.

\section{REFERENCES}

1. Manegin Yu.V., Pritomanov A.E., Spittel T., Knaushner A.M. Hot pressing of pipes and profiles. Moscow: Metallurgy. 1980, 272 p. (in Russian).

2. Hensel A., Spittel T. Calculation of energy-power parameters in the processing of pressure metals. Moscow: Metallurgy. 1982, 160 p. (in Russian).

3. Dyja Kh., Bergeman G.V., Sokolov S.F., Andreev V.V. An experimental study of the deformation resistance of steels and determination of the parameters of hardening-softening during hot pressure treatment. Materials Working by Pressure. Kramatorsk: DSEA, 2015, 2 (41). pp. 211-217. (in Russian).

4. Klimenko P.L. Hardening of steel and non-ferrous metals during cold and hot deformation: monograph. Dnepropetrovsk: Porogi. 2011. 187 p. (in Russian).

5. Firsova T.I. Methodology for estimating the parameters of hardening-softening of rheologically complex metals from plastometric curves. Materials Working by Pressure. Kramatorsk: DSEA, 2012, 1(3), pp. 65-68. (in Russian).

6. Tretyakov A.V., Zyuzin V.N. Mechanical properties of metals and alloys during pressure processing. Moscow: Metallurgy. 1973, 224 p. (in Russian).

7. Lotkov A., Grishkov V., Baturin A., et al. Yield stress and reversible strain in titanium nickelide alloys after warm abc pressing. Materials. 2019, 3258, 12, pp. 1-14. DOI: http://www.doi:10.3390/ma12193258

8. Medvedev M.I., Bespalova N.A., Tsarkov A.K. Methods for determining the main parameters of the pressing of pipes from hardly deformable steels and alloys. Metallurgy and mining industry. 2007, 3, pp. 56-59. (in Russian). (in Russian).

9. Plastic deformation of steels and alloys. Collection of scientific papers of MISiS. Moscow: MISiS. 1996, 460 p.

10. Shofman L.A. Fundamentals of the calculation of stamping and pressing processes. Moscow: Mashgiz. 1961, 399 p. (in Russian).

Медведев М. И. - д-р техн. наук, ст. науч. сотр. НМетАУ; E-mail: medvedev@metal-forming.org;

Андреев В. В. - канд. техн. наук, доц. НМетАУ;

E-mail: andreiev@metal-forming.org;

Фролов Я. В. - д-р техн. наук, проф., зав. каф. НМетАУ;

E-mail: frolov@metal-forming.org;

Бобух А. С. $\quad$ - канд. техн. наук, доц. НМетАУ;

E-mail: bobukh@metal-forming.org.

НМетАУ - Национальная металлургическая академия Украины, г. Днепр. 